\title{
A pausa na produção da fala com comprometimento neurológico
}

\author{
Jussara Melo Vieira \\ Plínio Almeida Barbosa \\ Maria Inês Pegoraro-Krook \\ Universidade de São Paulo
}

\section{Abstract}

Dysarthria is associated with a deficit in phonemic planning or paralysis of specific speech articulators, besides a frequent velopharyngeal impairment and expected prosodic alterations. The main goal of this work is to describe the speech rhythm of a user of a palatal lift protheses by analyzing the way she signals some linguistic boundaries when reading a short story. Pause durations were measured and pauses were classified into six syntactic categories. The correlation coefficient between categories and durations is $62 \%$, signaling that not all variance can be solely explained by syntax, but also by considering prosody as the interplay between linguistic knowledge and constraints of a biomechanical system. Despite her neuromotor impairment, the subject is able to use pauses to signal a prosodic hierarchy. 


\section{INTRODUÇÃO}

fala é um fenômeno cultural, exclusivamente humano,
somente possível por sua vida em sociedade (SAPIR, apud
DuBOIS et al., 1998).

Transmitir um pensamento é muito mais que concatenar fonemas. Sempre se recorre a parâmetros que estão além da estrutura segmental, nos planos fonético/fonológico, sintático/semântico da língua falada, como a prosódia, que lhes confere um colorido peculiar. Esse colorido pode ser, entre outros, a expressão de sentimentos, emoções e/ou idéias do falante.

A prosódia é um supra-segmento que possui dois aspectos: 1) produção, identificado pelos três parâmetros clássicos: a duração representada pela diferença de tempo entre dois eventos, a freqüência fundamental e a intensidade; 2) percepção, identificada pelas noções de duração percebida, altura e volume (BARBOSA, 1999).

Como a produção da prosódia está inserida na fala, como esta para que seja adequada, é necessária a integração do sistema nervoso central, da audição, do sistema respiratório, da laringe e das cavidades supraglóticas (PEGORARO-KROOK, 1995).

Do ponto de vista fisiológico-acústico, a fala tem origem na vibração das pregas vocais, provocada pelo ar expirado dos pulmões, produzindo um som (voz) que será modelado pelos articuladores (lábios, bochechas, mandíbula, dentes, palato duro, palato mole, paredes faríngeas) e ressonadores (regiões supraglóticas da laringe e faringe, cavidades oral e nasal), que compõem o trato vocal. O comprome-timento de quaisquer uma dessas estruturas pode resultar em prejuízo para a fala.

Os indivíduos com lesões neurológicas, congênitas ou adquiridas, podem apresentar disartria que é uma desordem neuromuscular com fraqueza, incoordenação, levando a uma perturbação da articulação dos fonemas devido ou a uma lesão central (lesão cortical), que causa 
um déficit na programação desses fonemas ou a lesões periféricas, que causam a paralisia de certos órgãos motores de execução da atividade de fala (DUBOIS et al., 1998). O tipo de disartria relacionase ao local e à extensão da lesão cerebral (BOONE \& PLANTE, 1994). Os músculos da língua, dos lábios e do palato mole podem estar afetados e apresentarem incoordenação, fraqueza, hipertonia ou hipotonia e a velocidade de fala pode estar diminuída conforme o grau de paresias e paralisias (ZIEGLER et al., 1988).

Pelo comprometimento neuromuscular, espera-se uma dificuldade em controlar a respiração, especialmente o fluxo expiratório no momento de fala, pois uma fadiga aparece conforme prolonga-se a fala, corroborando para uma incoordenação pneumo-fono-articulatória.

Também é freqüente a presença da incompetência velofaríngea (IVF) levando a uma ressonância hipernasal, pois o acoplamento entre as cavidades oral e nasal resulta em uma perda indesejada de ar (emissão de ar nasal) e de som durante a produção da fala, o que acarreta uma ressonância nasal excessiva, popularmente conhecida como fala fanhosa (PEGORARO-KROOK, 1995).

Problemas dessa natureza, não sendo tratados cirurgicamente, apresentam indicação para uso da prótese de palato elevadora, que consiste em um aparelho intra-oral removível com uma extensão (porção elevadora) feita de resina, a qual tem a finalidade de elevar o palato mole em direção à parede posterior da faringe (BÓ \& PEGORARO-KROOK, 2001). Portanto, o uso da prótese de palato elevadora é o tratamento mais indicado para a IVF, quando associada à presença de paralisias envolvendo os músculos do esfíncter velofaríngeo (TACHIMURA et al., 2000), a fim de estimular a função velofaríngea em associação com a terapia de fala (TACHIMURA et al., 2001).

Diante desses comprometimentos neurológicos que afetam a fala, tratados ou não proteticamente, a prosódia está alterada.

Assim, o objetivo do presente trabalho é descrever, em termos de produção da estruturação rítmica, a fala de uma jovem neurologicamente prejudicada que faz uso da prótese de palato elevadora, para determinar como ela assinala fronteiras lingüísticas ao longo dos enunciados de uma narrativa. 


\section{METODOLOGIA}

A participante desse estudo é a jovem I.M.M, regularmente matriculada no Hospital de Reabilitação de Anomalias Craniofaciais (HRAC) - USP/Bauru, do sexo feminino, com 26 anos e 3 meses de idade, na data de gravação (12/12/00), cujo comprometimento neurológico é em virtude de um traumatismo crânio-encefálico ocasionado por uma queda de uma mobilete. Por apresentar IVF, faz uso da prótese de palato elevadora desde 1995.

No Laboratório de Fonética Acústica do HRAC, sala tratada acusticamente, solicitou-se que a jovem, usando a prótese de palato elevadora, realizasse a leitura do texto exclusivamente composto por fonemas orais "A história do urso preto" (SUGUIMOTO \& PEGORAROKROOK, 1996) (Anexo 1). Essa leitura foi gravada em uma fita digital Sony, utilizando-se o Digital Audio Tape (DAT) DTC-690, também da marca Sony. Posteriormente, editou-se essa gravação via computador PC-IBM, por meio do programa WaveStudio (Creative Sound). A edição foi armazenada em disquete para zip drivee, então, analisada pelo Multi-Speech, modelo 3700, da Kay Elemetrics, da seguinte forma: trecho a trecho da leitura, com auxílio do espectrograma, fizeram-se marcações de duração de pausa (em milissegundos). As fronteiras das pausas foram assim determinadas: a primeira marcação correspondeu ao início da última vogal do segmento emitido antes da pausa, e a marcação final correspondeu ao início da primeira vogal do segmento seguinte (Fig. 1). A razão de uma tal marcação de pausa fundamenta-se nos trabalhos de Grosjean e colegas (1979, 1983), pois melhor revela a hierarquia das forças prosódicas subjacentes, o que é confirmado em Português Brasileiro (PB) no trabalho de Barbosa (1999), que ressalta, além disso, a importância do onset da vogal como delimitador por excelência de unidades prosódicas mínimas. 


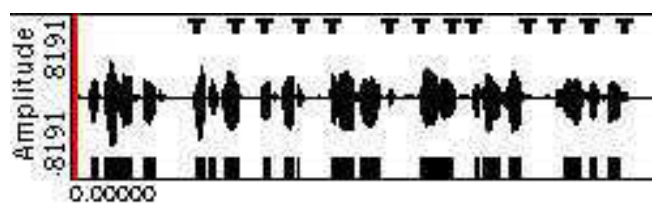

Figura 1 - Demonstração das marcações sobre os trechos da leitura do texto oral "A história do urso preto"

Após as marcações, estabeleceram-se categorias (Tab. 1), com base léxico-gramatical, para se caracterizar as fronteiras lingüísticas onde tais pausas aconteceram, na tentativa de se hierarquizar suas ocorrências. Quanto menor o número da categoria, maior a força da fronteira sintática por esse número representada e, conseqüentemente, maior o efeito da projeção dessa força sobre a cadeia da fala.

Tabela 1 - Descrição das categorias estabelecidas para esse estudo

\begin{tabular}{cl}
\hline \hline Categorias & Tipo de fronteira lingüistica \\
\hline \hline 1 & entre frases ((.)). \\
2 & entre orações, após introdutores e no início de conjunções. \\
3 & entre sintagmas nominal e verbal. \\
4 & no sintagma verbal, entre sintagma nominal e sintagma \\
& preposicional; ou entre o verbo e sintagma nominal ou \\
& preposicional. \\
5 & no início de sintagma preposicional dentro de sintagma \\
& no início de sintagma preprosicional dentro de sintagma \\
& preposicional dentro de sintagma adverbial. \\
\hline \hline
\end{tabular}

De posse das durações e suas categorias, por meio do aplicativo Excel (Microsoft, 2001), estabeleceram-se as relações entre elas e aplicou-se a análise estatística de correlação, sabendo-se que se a taxa de correlação for próxima ou maior que 70\%, mais da metade da variança dos dados pode ser explicada apenas pela assinalação de força de fronteira sintática via duração. 


\section{RESULTADOS E DISCUSSÃO}

Na Tabela 2, é possível verificar as durações encontradas, em ordem crescente, e as categorias estabelecidas.

Tabela 2 - Duração (ms), fronteiras lingüísticas e suas categorias, a partir da leitura do texto "A história do urso preto"

\begin{tabular}{cccc}
\hline Duração (ms) & $\begin{array}{c}\text { Fronteiras } \\
\text { Lingüisticas }\end{array}$ & Categorias & $\begin{array}{c}\text { Localização } \\
\text { no Texto* }\end{array}$ \\
\hline 62 & zoológico da & 6 & $\mathrm{~F} 1$ \\
271 & dias ele & 4 & $\mathrm{~B} 7$ \\
419 & vive atrás & 3 & $\mathrm{E} 2$ \\
498 & dia de & 5 & $\mathrm{D} 4$ \\
516 & aparece logo & 3 & $\mathrm{E} 1$ \\
543 & barulho das & 5 & $\mathrm{D} 3$ \\
557 & história do & 5 & $\mathrm{D} 2$ \\
560 & grades do & 5 & $\mathrm{D} 1$ \\
563 & dia, resolveu & 2 & $\mathrm{C} 5$ \\
566 & preso. Decidiu & 1 & $\mathrm{~A} 7$ \\
580 & cedo para & 4 & $\mathrm{~B} 6$ \\
584 & olhar os & 4 & $\mathrm{~B} 5$ \\
597 & floresta. Hoje & 1 & $\mathrm{~A} 6$ \\
600 & ver os & 4 & $\mathrm{~B} 4$ \\
601 & pássaros e & 2 & $\mathrm{C} 4$ \\
614 & descobriu que & 4 & $\mathrm{~B} 3$ \\
627 & viu que & 4 & $\mathrm{~B} 2$ \\
628 & jaula. Logo & 1 & $\mathrm{~A} 5$ \\
635 & triste, pois & 2 & $\mathrm{C} 3$ \\
636 & Decidiu que & 2 & $\mathrm{C} 2$ \\
637 & preto que & 2 & $\mathrm{C} 1$ \\
649 & esperar o & 4 & $\mathrm{~B} 1$ \\
704 & araras. Outro & 1 & $\mathrm{~A} 4$ \\
750 & bichos. Gosta & 1 & $\mathrm{~A} 3$ \\
768 & difícil. Ficou & 1 & $\mathrm{~A} 2$ \\
903 & cidade. Todos & 1 & $\mathrm{~A} 1$ \\
\hline \hline
\end{tabular}

* Em termos da Duração (ms): A>B $>\mathrm{C}>\mathrm{D}>\mathrm{E}>\mathrm{F} ; \mathrm{A} 1>\mathrm{A} 2>\mathrm{A} 3$ e assim por diante.

As categorias que mais ocorreram foram as de número 1 e 4 , com 7 ocorrências; seguidas das categorias 2, 5, 3 e $6 \operatorname{com}$ 5, 4, 2, e 1 ocorrências, respectivamente. 
Relacionando-se as categorias encontradas com as durações, obteve-se o Gráfico 1.

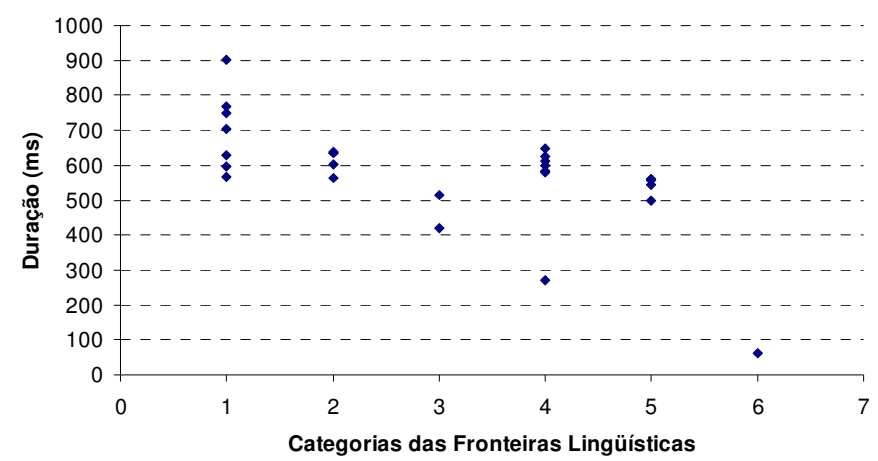

Gráfico 1 - Relação das categorias estabelecidas das fronteiras lingüísticas com sua duração, a partir da leitura do texto oral "A história do urso preto".

Analisando-se o gráfico, percebeu-se que a concentração na categoria 1 ocorre com maior freqüência nas maiores durações, delineando-se uma tendência de quanto mais fraca a categoria, menor a duração.

$\mathrm{Na}$ análise estatística de correlação, obteve-se o valor absoluto de 0,62 , o que corresponde a 62\%. Esta correlação é significativa, porém menor que $70 \%$. Isso assinala que nem toda a variança da duração pode ser explicada apenas pelas categorias sintáticas tais como escolhidas. Isso significa que elas precisam ser reavaliadas, notadamente com teorias sintáticas mais apropriadas, e que durações assinalam uma hierarquia prosódica que não é isomórfica com a hierarquia sintática, visto que a prosódia é o resultado da negociação entre um sistema lingüístico e um sistema biomecânico de produção de fala (BARBOSA, 1999).

A localização das pausas e sua duração possibilitaram o estabelecimento de sua relação com a saída de ar e a conseqüente fadiga respiratória, utilizando ar residual até o esgotamento do fôlego (FERREIRA, 1996), esperada em casos com este tipo de comprometimento neurológico. Ou seja, maior duração das pausas quanto mais esteja no final do texto lido. 
Pela existência de pausas e pelo estabelecimento de suas durações em relação às fronteiras lingüísticas, percebeu-se que, apesar de seus comprometimentos neuromotores, a jovem é capaz de fazer uso das pausas para estabelecer uma hierarquia prosódica. Percebe-se, então, que o estabelecimento das pausas tem mais relação com a estrutura da sentença (GROSJEAN, GROSJEAN e LANE, 1979) do que com a condição pneumo-fono-articulatória do falante, apesar de se esperar desobediência à pontuação de um texto pela incoordenação pneumo-fono-articulatória, como afirma Ferreira (1996, p. 11).

\section{CONCLUSÃO}

Para o estabelecimento do quadro lingüístico de um falante é preciso ter muito claro até que ponto a fisiologia responde por seu desempenho lingüístico, fazendo, da melhor forma possível, sua relação com os aspectos puramente lingüísticos, avaliando a qualidade e não apenas a quantidade de sua competência lingüística. Espera-se que aspectos lingüísticos como a prosódia apresentem-se deficitários, em nível de desempenho, mas podem estar íntegros do ponto de vista da competência. Perspectivas deste tipo merecem atenção, para que se conheça, cada vez mais, os prejuízos de fala de indivíduos com comprometimento neurológico; sua capacidade lingüística remanescente; as estratégias compensatórias de que utiliza; o quanto a parte neuromotora responde e corresponde à sua competência lingüística; como estão os outros aspectos prosódicos, como o ritmo, a entonação e a acentuação, visto que as pausas ocorreram. A partir disso, poderão ser propostos procedimentos terapêuticos mantenedores ou ampliadores das atuais habilidades lingüísticas, da participante deste estudo bem como avaliar o uso da prótese de palato elevadora com finalidade fisiológico-acústico-lingüística. Assim, serão propostos a este falante uma melhor qualidade de vida e uma maior compreensão de sua comunidade lingüística quanto à sua competência/desempenho lingüísticos. Sem esquecer que o aprofundamento na estrutura da língua se faz necessário, a fim de se proporem categorizações cada vez mais adequadas e, sem esquecer, ainda, que a percepção prosódica do ouvinte, quanto ao desempenho do falante, também deve ser investigada. 


\section{REFERÊNCIAS BIBLIOGRÁFICAS}

BARBOSA, P. A. Revelar a estrutura rítmica de uma língua construindo máquinas falantes: pela integração de ciência e tecnologia de fala. In: SCARPA, E. M. (Org.). Estudos de Prosódia, Campinas/SP: Editora da Unicamp, 1999. 327p. Cap. 1.

BELL-BERTI, F.; GELFER, C.E.; BOYLE, M. Utterance-final lengthening: the effect of speaking rate. ICPhS 95, Stockholm, 1, sessão 8.3, p.162-5, 1995.

BÓ, F. R.; PEGORARO-KROOK, M. I. Prótese de Palato. Disponível em: <<http:/ /www.fob.usp.br/depart/baf/protesedepalato/oque.htm>>. Acesso em: 11/12/ 2001.

BOONE, D.R.; McFARLANE, S.C. A voz e a terapia vocal. 5. ed. Trad. Sandra Costa. Porto Alegre: Artes Médicas, 1994. 300p.

CRYSTAL, T.H.; HOUSE, A.S. Segmental durations in connected speech signals: preliminary results. Journal of the Acoustical Society of America, n. 72, v. 3, p.705-16, set. 1982 .

DUBOIS, J.; GIACOMO, M.; GUESPIN, L.; MARCELLESI, C.; MARCELLESI, J.B.; MEVEL, J-P. Dicionário de Lingüística, 10. ed. São Paulo: Editora Cultrix, 1998.

FANT, G.; KRUCKENBERG, A. Quantal theory of speech timing. Fonetik 96, Swedish Phonetics Conference, Nässlingen, p. 41-44, maio 1996.

FERREIRA, L.P. Respiração: tipo, capacidade e coordenação pneumo-fonoarticulatória. In: FERREIRA et al. Temas de Fonoaudiologia. São Paulo: Loyola, 1996. Cap.1, p.11.

FOWLER, C. Production and perception of coarticulation among stressed and unstressed vowels, Journal of Speech and Hearing Research, n. 47, p. 127-139, 1981, apud BARBOSA, P.A. Revelar a estrutura rítmica de uma língua construindo máquinas falantes: pela integração de ciência e tecnologia de fala. In: SCARPA, E.M. (Org.). Estudos de Prosódia. Campinas/SP: Editora da Unicamp, 1999. 327p. Cap. 1.

GROSJEAN, F. How long is the sentence? Prediction and prosody in the on-line processing of language. Linguistics, n. 21, p.501-529, 1983.

GROSJEAN, F.; GROSJEAN, L.; LANE, H. The patterns of silence: performance structures in sentence production. Cognitive Psychology, n. 11, p.58-81, 1979.

MONNIN, P.; GROSJEAN, F. Mémoires originaux. L'Année Psychologique, n. 93, p.9-30, 1993. 
NOOTEBOOM, S.G. How far do we look ahead while speaking? ICPhS 95, Stockholm, 4, sessão 86.2, p.578-81, 1995.

PEGORARO-KROOK, M.I. Avaliação da fala de pacientes que apresentam inadequação velofaringea e que utilizam prótese de palato. 1995. 130p. Tese (Doutorado) - Escola Paulista de Medicina, Universidade Federal de São Paulo, São Paulo.

SAPIR, E. apud DUBOIS, J.; GIACOMO, M.; GUESPIN, L.; MARCELLESI, C.; MARCELLESI, J.B.; MEVEL, J-P. Dicionário de Lingüistica, 10. ed. São Paulo: Editora Cultrix, 1998.

SCOTT, D. R. Duration as a cue to the perception of a phrase boundary a), $J$. Acoust. Soc. Am., n. 71, v. 4, p. 996-1007, abr. 1982.

STREETER, L. A. Acoustic determinants of phrase boundary perception. J. Acoust. Soc. Am., n. 64, v. 6, dez. 1978.

SUGUIMOTO, M.L.C.P.; PEGORARO-KROOK, M.I. Avaliação nasométrica em adultos normais falantes do português brasileiro. Pró-Fono: Revista de Atualização Científica, n. 8, v. 1, p. 7-12, 1996.

TACHIMURA, T.; NOHARA, K.; HARA, H.; WADA, T. Effect of placement of a speech appliance on levator veli palatini muscle activity during speech. Cleft Palate-Craniofacial Jounal, v. 37, n. 5, p. 478-482, set. 2000.

TACHIMURA, T.; NOHARA, K.; FUJITA, Y.; HARA, H.; WADA, T. Change in levator veli palatini muscle activity of normal speakers in association with elevation of the velum using an experimental palatal lift prosthesis. Cleft Palate-Craniofacial Jounal, v. 38, n. 5, p. 449-454, set. 2001.

ZIEGLER, W.; HOOLE, P.; HARTMANN, E. D. Von Cramon Accelerated speech in dysarthria after acquired brain injury: Acoustic correlates. British Journal of Disorders of Communication, n. 23, p. 215-228, 1988. 


\section{ANEXO 1}

\section{A história do urso preto}
D2
C1
A6
E2

Esta é a história do urso preto que veio da floresta. Hoje ele vive atrás

$\begin{array}{lllll}\text { D1 } & \text { F1 } & \text { A1 } & \text { B7 } & \text { E1 }\end{array}$

das grades do zoológico da cidade. Todos os dias ele aparece logo

$\begin{array}{lllll}\text { B6 } & \text { B4 } & \text { A3 } & \text { B5 }\end{array}$

cedo para ver os outros bichos. Gosta de olhar os pássaros e de ouvir

$\begin{array}{lllll}\text { D3 } & \text { A4 } & \text { C5 } & \text { A5 } & \text { B2 }\end{array}$

o barulho das araras. Outro dia, resolveu sair da jaula. Logo viu que
A2
$\mathrm{C} 3$
B3
A7
C2

era difícil. Ficou triste, pois descobriu que estava preso. Decidiu que

B1 D4

deveria esperar o dia de sua verdadeira liberdade. 